\title{
Tuberculosis in alpaca (Lama pacos) on a farm in Ireland. 2. Results of an epidemiological investigation
}

\author{
Connolly, D.J. ${ }^{1}$, Dwyer, P.J. ${ }^{2}$, Fagan, J. ${ }^{2}$, Hayes, M. ${ }^{3}$, Ryan, E.G. ${ }^{4}$, Costello, E. ${ }^{5}$, Kilroy, A. ${ }^{6}$ and More, S.J..$^{4,7}$ \\ ${ }^{1}$ Ballybaun, Gort, Co. Galway, Ireland \\ 2 Department of Agriculture, Fisheries and Food, Regional Veterinary Laboratory, Athlone, Co. Westmeath, Ireland \\ 3 Department of Agriculture, Fisheries and Food, Government Offices, Davis Street, Tipperary, Co. Tipperary, \\ Ireland \\ ${ }^{4}$ Section of Herd and Veterinary Public Health, UCD School of Agriculture, Food Science and Veterinary Medicine, \\ University College Dublin, Belfield, Dublin 4, Ireland \\ ${ }^{5}$ Department of Agriculture, Fisheries and Food, Central Veterinary Research Laboratory, Backweston Campus, Co. \\ Kildare, Ireland \\ ${ }^{6}$ Department of Agriculture, Fisheries and Food, Kilrush Road, Ennis, Co. Clare, Ireland \\ 7 Centre for Veterinary Epidemiology and Risk Analysis, UCD School of Agriculture, Food Science and Veterinary \\ Medicine, University College Dublin, Belfield, Dublin 4, Ireland
}

\begin{abstract}
Tuberculosis (TB), due to infection with Mycobacterium bovis was diagnosed in a flock of alpaca in Ireland in 2004. An epidemiological investigation was conducted to identify the risk of TB for farmed alpaca where TB is endemic, the origin of the infection, the potential for alpaca-to-alpaca transmission and appropriate control measures. The investigation focused on the alpaca flock (including the farm, animal movements and breeding, feeding and flock health practice), the disease episode (including animal disease events and subsequent control measures) and TB infection risk in the locality. The TB risk to alpaca is high in areas where infection is endemic in cattle and badgers and where biosecurity is inadequate. It is most likely that the source of infection for the alpaca was a local strain of $M$. bovis, present in cattle in this area since at least 2001 . Genotyping of isolates identified a single variable number tandem repeat (VNTR) profile in both cattle and alpaca in this region. Although a tuberculous badger was also removed from the vicinity, bacterial isolation was not attempted. On this farm, infection in alpaca was probably derived from a common source. Alpaca-to-alpaca transmission seems unlikely. Two broad control strategies were implemented, aimed at the rapid removal of infected (and potentially infectious) animals and the implementation of measures to limit transmission. Tests that proved useful in detecting potentially-infected animals included measurement of the albumin-to-globulin ratio and regular body condition scoring. Skin testing was time consuming and unproductive, and early detection of infected animals remains a challenge. The flock was managed as a series of separate groupings, based on perceived infection risk. No further TB cases have been detected.
\end{abstract}

Key Words: alpaca, bovine tuberculosis, control, epidemiology, investigation, Lama pacos, Mycobacterium bovis, transmission

Corresponding author:

Dónal Connolly

Ballybaun,

Gort,

Co. Galway,

Ireland

Email: djconnolly@iol.ie

\section{Introduction}

Cases of tuberculosis (TB) in alpaca in their natural habitat in South America are believed to be rare, even though tuberculosis is endemic in local cattle, sheep and humans (Fowler, 1998). However, there are reports of infection in camelids (llama, alpaca, vicuña and guanaco) in South America held in close association with other infected livestock (Fowler, 1998). Mycobacterium bovis has been diagnosed in alpaca grazing with infected cattle in Argentina (R.P. Lopetequi, personal communication, 2008) and in alpaca (Barlow et al., 1999) and llama (Twomey et al., 2007) in the UK.

TB was recently reported in farmed alpaca in Ireland. This disease was suspected on clinical evaluation, and subsequently confirmed in a number of animals on post $\underline{\text { Irish Veterinary Journal }}$ Volume 61 Number 8 533-537, 2008

mortem examination. The causative agent was identified as Mycobacterium bovis (Ryan et al., 2008). However, a number of important questions had not been addressed at the time of diagnosis. Therefore, during a subsequent epidemiological investigation, as described here, the authors have sought to determine: (a) the TB infection risk for alpaca farmed in areas where TB is endemic; (b) the origin of the infection; (c) the potential for alpaca-toalpaca transmission; and (d) appropriate control measures, including the efficacy of predictor tests.

\section{Materials and methods}

An epidemiological investigation was arranged involving a private veterinary practitioner (PVP) and a veterinary research officer (VRO) of the Athlone Regional Veterinary 


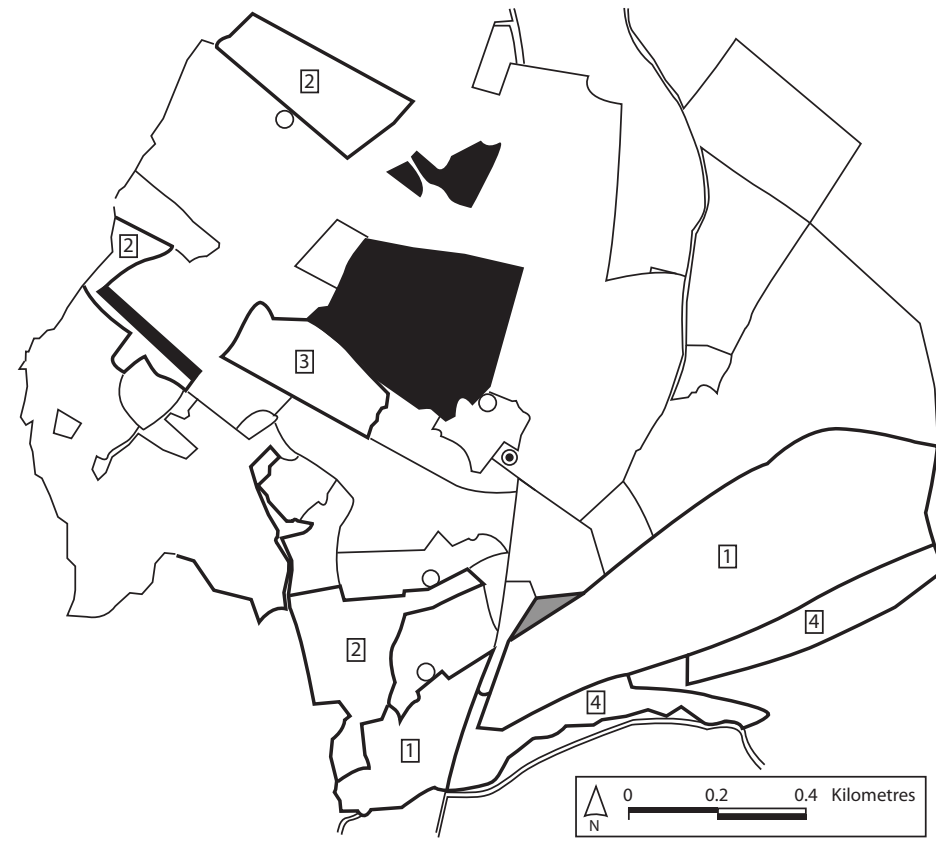

Fig 1: A map of the index and neighbouring farms. The index farm, with three distinct fragments is presented in solid black. All neighbouring land fragments are outlined, and neighbouring farms 1 to 4 are labelled. Alpaca grazed a field on neighbouring farm 1 (grey shading) during November 2003 to March 2004. The location of badge setts (open circle) and of an infected dead badger (in January, circle with dot) is shown.
Laboratory (ARVL), in conjunction with a group of final year veterinary undergraduates from the University Veterinary Hospital (UVH) under the direction of clinical staff, and with veterinary inspectors from the local District Veterinary Office, with area responsibility for scheduled and notifiable disease. The investigation focused on:

- the alpaca flock, including the farm (location, fragmentation, boundaries, fencing, opportunities for animal contact), animal movements and breeding, feeding and flock health practices;

- the disease episode, including animal health events and subsequent control measures; and

- TB infection risk in the locality.

Following initial diagnosis, a range of predictive diagnostic tests were used in the flock, including the tuberculin test and the lymphocyte proliferation test (Ryan et al., 2008). Tuberculin testing was conducted, following the initial on-farm investigation, at approximately 120-day intervals. Initially, the single intradermal comparative cervical skin tuberculin (SICCT) test was performed, using standard M. bovis and M. avium purified protein derivative (bovine and avian PPD, respectively). Later, following the recommendation of Frost (1999), the single intradermal comparative axillary tuberculin (SICAT) testing was preferred. Body condition scoring was done in association with tuberculin testing. Blood testing, to assess the albumin: globulin (A:G) ratio, was also regularly carried out: animals with A:G values greater or equal to 1.0 were treated as normal; those with $\mathrm{A}: \mathrm{G}$ between 0.7 and 0.9 were checked for weight loss, for respiratory signs and reaction at tuberculin testing. Those with A:G less than or equal to 0.6 were considered to be at high risk and isolated. Infection risk on neighbouring farms and in wildlife was determined after reviewing records held by the national Department of Agriculture, Fisheries and Food. In Ireland, TB testing data are available (all cattle are tested annually using the SICCT). Furthermore, the TB status of captured badgers (as part of the national TB programme) is available, based on post mortem examination.

$M$. bovis was isolated from samples using methods described previously (Ryan et al., 2008). VNTR typing (based on six VNTR loci: QUB11a, QUB11b, ETRA, MIRU26, VNTR4052 and VNTR1895) was conducted using primers and PCR conditions as described by Roring et al. (2004).

\section{Results}

\section{The alpaca flock}

a. The farm

The farm is located in Ireland. The farm is almost 50 acres (19.5 hectares) in area, including a 40 -acre ( 16.2 hectares) plot and two non-contiguous fragments of three acres (1.2 hectares) and five acres (2.0 hectares, in two adjacent fields) (Figure 1). The 40-acre (16.2 hectares) plot, purchased and reclaimed during 2003-2004 from rough pasture with rocky outcrops, was not used by farmed stock for some years prior to reclamation. Each section is subdivided into a number of paddocks. The boundaries are double fenced, with sheep wire fencing placed a metre inside a traditional dry stone wall structure, which had partially collapsed in some places. Fencing ensured that contact with cattle was limited, except at one boundary point where a water trough was shared with cattle, but the fencing was not adequate to restrict movement of badgers. Contact by the alpacas with each other within their land section was routinely close. In addition, prolonged contact with cattle occurred on a few occasions when alpacas were grazed, at times of grass shortage, on separate lands (neighbouring farm 1) owned by one of the alpaca co-owners. A water trough was shared on the three-acre fragment between alpaca and cattle from neighbouring farm 2 (Figure 1). Prior to the outbreak, water and feeding troughs were placed at ground level, and were accessible to wildlife, including badgers.

b. Animal movements 
In May 2000, the flock was first established with the importation of 15 adult females and one juvenile male from Peru and Chile, via a farm in Northern Ireland after being quarantined in Belgium. All animals were negative on SICCT test for tuberculosis and were quarantined before and after entry. In 2002, three females were sold to another farm in west Ireland, and nine animals, each negative on the tuberculin test, were imported from Northern Ireland from a flock whose owners had returned to Australia. In 2003, four tuberculin test negative animals were imported from Australia; three adult females and one juvenile male. In 2004, of the three alpacas sold to the above-mentioned farm in west Ireland, two females were returned showing weight loss, one with a cria (juvenile alpaca) at foot and a third adult female, having lost weight, had died on this farm without post mortem examination. Additionally in 2004, two females, one with cria at foot, were introduced for four weeks for breeding purposes from a farm in south Ireland. From November 2003 to March 2004, a number of weanlings (including animals D, E, F and G) and one pregnant adult were moved to fields (on neighbouring farm 1) managed by one of the alpaca co-owners.

c. Breeding, feeding and flock health practices Females were bred when 15-16 months old and yearly thereafter. Cria were weaned when five to six months old and separated by sex when animals were one year in age. At this time, the young females were returned to the main flock. The juvenile males were kept separately. The alpaca were kept on a ration modulated to achieve a body condition score of less than or equal to three. All animals were kept continuously at grass throughout the year, and supplemented with a commercial horse crunch at $18 \%$ protein, and forage (hay) as necessary. Trace element supplementation with selenium injection was introduced in 2003. In early 2004, there were approximately 60 alpaca on the farm. Anthelmintics and vaccinations (against clostridial disease) were administered routinely, as per schedules recommended for sheep. Teeth were filed and feet trimmed periodically.

\section{The disease episode}

\section{a. Animal health events}

Following importation in May 2000, one cria was lost in December due to a 'chill'. The flock was initially fed very little with the aim to improve coat fibre and, by January 2001, some animals were in poor body condition and were weak. Two animals died, one from pneumonia and the other had lesions compatible with chronic liver fluke infestation. There was no evidence, at gross post mortem, of TB in either case.

In May 2002, the alpacas were successfully treated for an ectoparasitic skin condition. In June 2002, an alpaca showed signs of pneumonia with weight loss, weakness and a temperature of $40^{\circ} \mathrm{C}$. She was treated with antibiotics, but died the following morning without post mortem examination.

In March of 2003, an animal was found recumbent with weight loss and tenesmus, and died a few hours later. Post mortem examination carried out at the Limerick Regional Veterinary Laboratory (LRVL) attributed death to fluke infestation with sarcocystosis also evident. In May 2003, two crias two to three weeks in age died. At post mortem examination at LRVL, these losses were histologically attributed to a vitamin E/selenium cardiomyopathy. As a result, trace element supplementation was introduced. In 2004, rickets in young alpacas was diagnosed. In May of that year, an adult full-term pregnant female alpaca died suddenly. Post mortem examination revealed acute pneumonia with copious exudate present, but no lesions of TB were noted. In July 2004, the offspring of this alpaca died at pasture with weight loss evident over the previous few weeks. No post mortem examination was carried out. In September 2004 an adult female died suddenly at pasture. Again, no post mortem examination was carried out. In late October 2004, two adult females, D and E, presented with mild respiratory symptoms. Tuberculosis was suspected on clinical grounds and confirmed on post mortem examination (Ryan et al., 2008).

In late 2004, another alpaca, (F aged 21/4 years,) presented with mild respiratory signs. Following euthanasia in January 2005 , TB due to $M$. bovis was diagnosed on post mortem examination. In February 2005, a female alpaca, G, died suddenly with miliary TB evident on post mortem examination. Miliary tuberculosis was diagnosed in a further alpaca, H, in April 2005.

No further cases of tuberculosis have been diagnosed since April 2005.

VNTR typing was conducted on $M$. bovis isolates from four alpaca (animals D, E, F and G). The VNTR profile identified in each case was 1028 544 (loci sequence QUB11a, QUB11b, ETRA, MIRU26, VNTR4052, VNTR1895).

\section{b. Control measures}

Complete depopulation was recommended, but not undertaken by the flock owners. Subsequently, disease management focused on efforts to prevent further entry of disease (through attention to farm biosecurity and further animal purchases) and to prevent within-flock transmission, including the early identification and removal of infected animals and the formation and management of specific 'risk' groups.

An initial assessment of the infection status of apparentlynormal animals in the flock was conducted in February 2005, based on age and signalment, blood testing (A:G ratio less than one), results of a tuberculin test conducted in January 2005, history and clinical signs and history of high-risk contacts (with known infected animals). A total of 10 animals were considered at high risk. Subsequently, diagnostic efforts focused on tuberculin testing, blood testing and body condition scoring. Tuberculin testing was repeated in June 2005 using a single cervical injection of bovine PPD, as recommended by the New Zealand camelid voluntary control programme (Alpaca Association New Zealand, 2004). Four of 63 animals were deemed 
positive, including two that were subsequently euthanased. However, there was no evidence of tuberculosis based on gross post mortem and lymph node histopathology and culture. Further whole-herd tuberculin testing was conducted in October 2005 and January 2006, but no reactors were found. A:G ratios were measured in selected animals in November 2004 and February 2005, and on all animals in April and July 2005. The lymphocyte proliferation test was conducted in April 2005 on 10 animals identified at high infection risk, but with inconclusive results. Body condition scoring was conducted on nine occasions between December 2004 and January 2006. Protective clothing, including face masks, was worn during handling, and strict disinfection was practised. These data were used to guide management strategies. High risk animals were managed separately from the main flock.

\section{$T B$ infection risk in the locality}

Bovine TB is relatively common in the locality. Of the 12 farms immediately contiguous to the index farm, two had experienced a TB episode during 2004 (neighbouring farm 1 experienced 21 reactors during August to December; neighbouring farm 2 experienced four reactors during June to September). VNTR typing was conducted on M. bovis isolates from nine reactor animals identified in 2004 from neighbouring farm 1; a single VNTR profile was identified. This profile was the same as that identified in the four alpaca from the index farm. Additional TB outbreaks were detected on neighbouring farm 3 in 2005 (one reactor in December) and neighbouring farm 4 in 2006 (six reactors during June to August) (Figure 1).

At this stage, this $M$. bovis VNTR profile has not been identified in M. bovis isolates from any other Irish county. In addition to the isolates from the index farm (alpaca) and neighbouring farm 1 (cattle), strains with this VNTR profile have been isolated in two additional circumstances: from a 1999-born steer raised on two farms prior to slaughter during July 2001 (for six months on a farm 56 $\mathrm{km}$ from the index farm, for the remaining 23 months on a farm $10 \mathrm{~km}$ from the index farm), and from four cattle slaughtered in 2005 from a farm $6.5 \mathrm{~km}$ from the index farm).

In January 2005, a dead badger was found near the index farm. Although tuberculous lesions were detected grossly at post mortem, culture was not attempted. A number of badger setts, some intermittently active, are located in the vicinity of the index farm (Figure 1) and there was on-farm badger activity as evidenced by digging. The removal of badgers, under national licence, commenced in early 2005. In total, one of seven $(14.2 \%)$ captured badgers was TB positive.

\section{Discussion}

\section{TB infection risk among alpaca}

Contrary to published information, alpaca appear susceptible to infection with M. bovis. At post mortem, spectacular pathological changes in infected animals, including severe diffuse granulomatous bronchointerstitial pneumonia were noted. As discussed below, TB is a significant problem in this locality. Although TB among alpaca is considered rare (Fowler, 1998), we believe this is a consequence of non-exposure rather than low susceptibility.

\section{The source of infection}

In this outbreak, it is most likely that the source of infection for the alpaca was a local strain of M. bovis, present in cattle in this area since at least 2001. The VNTR profile in alpaca was identical to that identified in strains isolated from reactor cattle as early as 2001 on three different farms in the general locality. Furthermore, TB was initially detected among a sub-group of alpaca (including animals D, E, F and G) that grazed a field on neighbouring farm 1 (managed by a co-owner of the alpaca) during a period of grass scarcity during November 2003 to March 2004. A substantial TB breakdown, with 21 reactors, was first detected on neighbouring farm 1 in August to December 2004 and on the index farm (with alpaca D) on October 2004. It is likely that at least four alpaca were infected during this period. Badgers are an important contributor to the epidemiology of tuberculosis of cattle in Ireland (Griffin et al., 2005; More and Good, 2006). Infected badgers were found in the locality and badger activity was detected on the index farm, but $M$. bovis isolation (and therefore VNTR profiling) was not attempted. It is very unlikely that imported alpaca were the source of infection. This would only be possible if the original alpaca had been infectious soon after arrival in May 2000 and infection was subsequently disseminated (to a distance of at least 10 $\mathrm{km}$, presumably by wildlife) some months prior to July 2001, when the first case with this VNTR profile was slaughtered.

\section{The potential for alpaca-to-alpaca transmission}

TB was confirmed in five alpaca (D, E, F, G and H) during this outbreak, resulting in a within-flock incidence of approximately $10 \%$ between late 2004 and early 2005 . In each case, miliary tuberculosis was diagnosed, with multiple organ involvement including lung. Throughout this investigation, the authors sought to determine, following introduction into the flock, whether infection was subsequently disseminated through alpaca-to-alpaca transmission. The evidence remains uncertain. On the one hand, pathology was severe and respiratory excretions were likely to be infectious. Hence, the authors' attention to personal safety and the decision to manage the flock in groupings based on risk. Within each grouping, the alpaca were in close contact, particularly during handfeeding. On the other hand, however, the outbreak should have continued, and likely intensified, if alpaca-to-alpaca transmission was important. The temporal pattern of presentation (all cases with advanced pathology over a relatively short period) is more suggestive of a common source (rather than a propagating) epidemic. 


\section{Control measures}

Control measures were implemented in this flock with two key objectives: the rapid removal of infected (and potentially infectious) animals and implementation of strategies to limit transmission, both alpaca-to-alpaca and to alpaca from cattle and badgers. To this point, it has been very difficult to assess the success of these measures. No cases have been detected since April 2005.

A range of methods were used to detect infected animals, including tuberculin testing, measurement of A:G ratios and body condition scoring. Skin testing was technically demanding, and the results were difficult to interpret. The IFN- $\gamma$ test has not been validated for use as a diagnostic test for TB in camelids (Cousins and Florissen, 2005; Buick, 2006). Because hypoalbuminaemia and hyperglobulinaemia were marked among the case animals, the A:G ratio proved a useful prognostic indicator. Hypoalbuminaemia is attributed to a number of mechanisms, including chronic inflammation, malnutrition, liver disease, protein losing nephropathy and protein losing enteropathy. Hyperglobulinaemia occurs in chronic inflammatory processes due to prolonged antigenic stimulus and is accompanied by a decrease in blood albumin levels (Keshgegian, 1984). Inflammation is associated with a greater fractional catabolic rate of albumin and, when extreme, increased transfer of albumin out of the vascular compartment (Don and Kaysen, 2004). In cases D and E, the $A: G$ ratios were 0.53 and 0.59 , respectively, during the latter stage of illness. The differing results from case $\mathrm{D}$ on two occasions ( 0.22 from ARVL, 0.53 from UVH) is a reflection of the severity of the more advanced catabolic state on presentation to the UVH which had lead to an end stage drop in all protein fractions (total protein, albumin and globulin) in the blood. Although non-specific, body condition scoring was also a very useful indicator of general health among animals in this flock.

A range of options for flock management were considered, but some proved impractical. The flock was managed as a series of separate groupings, based on perceived infection risk. This strategy was constrained by the above-mentioned difficulties associated with the early detection of infected animals. Under national licence, focused badger culling is being conducted. However, the cost of badger-proof fencing was prohibitive.

\section{Conclusions}

In conclusion:

- This infection was very unlikely to be related to alpaca importation, noting the interval between importation and disease detection, and the presence of an identical $M$. bovis VNTR profile in local cattle.

- At least four alpaca became infected whilst grazing on farm 1 . The cattle on this farm, which was owned by a co-owner of the alpaca, were infected with an identical M. bovis VNTR profile that had first been identified in local cattle in 2001. Farm-to-farm dissemination of this VNTR profile may have occurred as a result of the movement of infected badgers.
- There is little evidence in support of alpaca-to-alpaca transmission, noting that no new cases have been detected. Cousins and Florisson (2005) suggest that alpaca are not a maintenance host; rather infection is transmitted via contact with other infected animals.

- Alpaca are susceptible to TB, and pathology can be severe. The TB risk to alpaca is high in areas where infection is endemic, and where biosecurity to prevent entry of infection is inadequate. In relevant countries, this will present significant challenges to successful alpaca production.

\section{Acknowledgements}

We thank Daniel Collins for his assistance with mapping and with interrogation of the national database and Joanne McLernon for VNTR typing of isolates.

\section{References}

Alpaca Association New Zealand (2004). Disease control: camelid voluntary tuberculosis control scheme. Farm Network AHB 13. http:// www.alpaca.org.nz/downloads/tb-scheme-010404.pdf. Accessed on 07 August 2007.

Barlow, A.M., Mitchell, K.A. and Visram, K.H. (1999). Bovine tuberculosis in llama (Lama lama) in the UK. Veterinary Record 145: 639-640. Buick, W. (2006). TB in domestic species other than cattle and badgers. Government Veterinary Journal 16: 87-91.

Cousins, D.V. and Florisson, N. (2005). A review of tests available for use in the diagnosis of tuberculosis in non-bovine species. Revue scientifique et technique - Office International des Epizooties 24: 1039-1059.

Don, B.R. and Kaysen, G. (2004). Serum albumin: relationship to inflammation and nutrition. Seminars in Dialysis 17: 432-437.

Fowler, M.E. (1998). Medicine and surgery of South American camelids, $2^{\text {nd }}$ Edition. Iowa State University Press, Ames.

Frost, B. (1999). Research update on diagnostic tests for tuberculosis in llamas/alpacas. Proceedings of the $102^{\text {nd }}$ Annual Meeting of the United States Animal Health Association, Minneapolis, Minnesota, USA. 3-9th October 1999.

Griffin, J.M., Williams, D.H., Kelly, G.E., Clegg, T.A., O’Boyle, I., Collins, J.D. and More, S.J. (2005). The impact of badger removal on the control of tuberculosis in cattle herds in Ireland. Preventive Veterinary Medicine 67: 237-266

Keshgegian, A.A. (1984). Hypoalbuminemia associated with diffuse hypergammaglobulinemia in chronic diseases: lack of diagnostic specificity. American Journal of Clinical Pathology 81: 477-81.

More, S.J. and Good M. (2006). The tuberculosis eradication programme in Ireland: A review of scientific and policy advances since 1988. Veterinary Microbiology 112: 239-251.

Ryan, E.G., Dwyer, P.J., Connolly, D.J., Fagan, J., Costello, E. and More, S.J. (2008). Tuberculosis in alpaca (Lama pacos) on a farm in Ireland. 1. A case report. Irish Veterinary Journal 61 (8): 527-531.

Roring, S., Scott, A.N., Hewinson, R.G., Neill, S.D. and Skuce, R.A. (2004). Evaluation of variable number tandem repeat (VNTR) loci in molecular typing of Mycobacterium bovis isolates from Ireland. Veterinary Microbiology 101: 65-73.

Twomey, D.F., Crawshaw, T.R., Anscombe, J.E., Farrant, L., Evans, L.J., Mcelligott, W.S., Higgins, R.J., Dean, G., Vordermeier, M., Jahans, K. and de la Rua-Domenech, R. (2007). TB in llamas caused by Mycobacterium bovis. Veterinary Record 160: 170. 